\title{
Plasma-arc Welding Sound Signature for On-line Quality Control
}

\author{
Yaowen WANG and Pengsheng ZHAO \\ Materials Science \& Engineering Department, Taiyuan University of Technology, Taiyuan 030024 China. \\ E-mail:wyw311@263.net
}

(Received on August 11, 2000; accepted in final form on November 16, 2000)

\begin{abstract}
On-line quality control in automated welding operations is an important factor contributing to higher productivity, lower costs and greater reliability of the welded components. The sound signal of plasma arc welding was acquired at high speed and investigated with the aid of computers. It is shown that the amplitude of the sound signal greatly varies with the variation of the status of the weld pool. The analysis of frequency domain indicated that the area below the curve of the low frequency band of the power density spectra, which may reflect the oscillation properties of the weld pool, is largest on the transition phase. The high frequency dominant peaks of the sound signal, which may relate to the plasma jet pulsation, is highest on the keyhole-formed phase. The method for detecting the behaviors of the weld pool keyhole was developed. The experimental results indicate that the acoustic emission is a usable, practical information source in the penetration quality detection of plasma arc welding.
\end{abstract}

KEY WORDS: plasma arc welding; power spectrum; sound signature; process control.

\section{Introduction}

Since the energy density and gas velocity in plasma arc are high, plasma arc welding can be performed in the keyhole mode. Plasma arc welding is extensively used to weld carbon steel, stainless steel, nickel-based alloys, titanium alloys and aluminum and magnesium alloys. ${ }^{1-3)}$ The keyhole stability in plasma arc welding is a crucial problem to achieve good quality welds. The variation of the keyhole status is reflected in the process emissions, such as sound and electromagnetic waves. By the investigation of the sound signature, it is possible to detect the behaviors of the keyhole in the weld pool during welding.

Recently, considerable effort has been directed toward the development of on-line sensing and significant progress has been made in gas metal arc welding, ${ }^{4-7)}$ laser beam welding, ${ }^{8,9)}$ and tungsten arc welding, et $a{ }^{10)}$ However, there is less research on the plasma arc welding. Techniques such as the efflux plasma ${ }^{11,12)}$ at the back of the welds, the light spectrum ${ }^{13)}$ and image sensing ${ }^{14)}$ on the front side of the welds have been proposed for on-line detection and quality control of the plasma arc welding. But, it is difficulty to reach the back of the welds in the welding of complex structures and a complex and high cost spectrometer is required, so that their applications are limited. Futamata, et al., ${ }^{15)}$ analyzed the plasma jet sound, but during plasma-arc keyhole welding the transferred arc is employed. Manz ${ }^{16)}$ deemed that the plasma arcs could sound like jet engines, and did not further investigate.

In the present work, the investigation of the sound signature of transferred plasma-arc keyhole welding is presented. The properties of the sound signal were analyzed in time domain and frequency domain. Algorithms are developed that are able to detect the status of the plasma-arc keyhole welding.

\section{Experimental}

A schematic drawing of the experimental configuration is shown in Fig. 1. The power supply is an inverter designed for plasma arc welding. The sound signal during welding was measured with a condenser microphone with a bandwidth of $20 \mathrm{~Hz}$ to $20 \mathrm{kHz}$, which was mounted near the welding torch. The sampling rate of the sound signal was $50 \mathrm{kHz}$.

In the experiments, a phototriode was attached to the plasma torch and positioned on the back of the weld so as to obtain the light signal of the efflux plasma and offer a reference signal of the keyhole states. The light signal together with the sound signal was simultaneously sampled and transferred for permanent storage to the computer Pentinum II.

Pure argon was used as the shielding gas and the plasma gas. The flow rate of the shielding gas was $15 \mathrm{l} / \mathrm{min}$ for the weld face. The flow rate of the plasma gas and the welding speed was $1.5 \mathrm{l} / \mathrm{min}$ and $320-330 \mathrm{~mm} / \mathrm{min}$. The welding

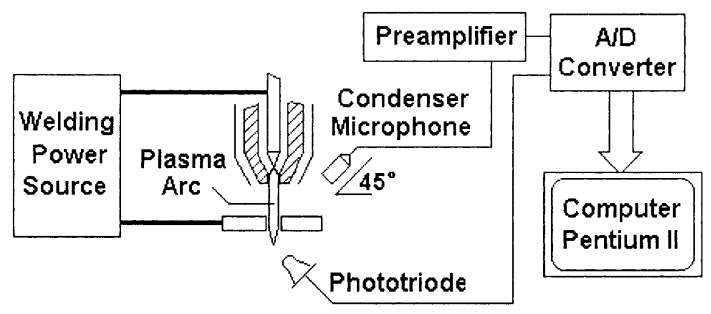

Fig. 1. Schematic drawing of the experimental system. 


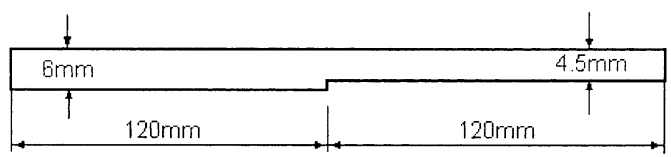

Fig. 2. The specimen employed in the experiments (front view).

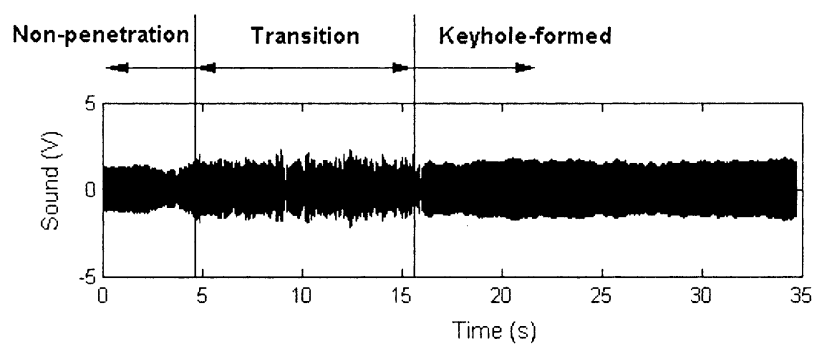

(A)

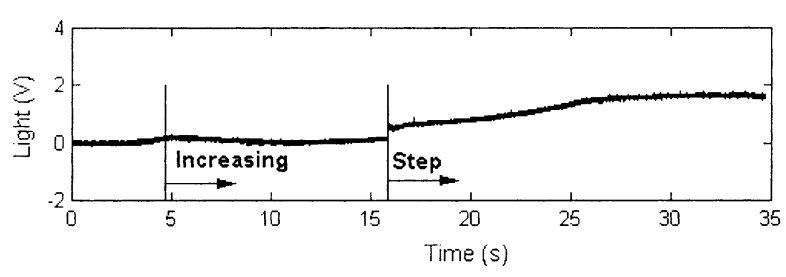

(B)

Fig. 3. The variation over time of the sound signal. A-The sound emission; B-The reference light signal.

current ranged from 130 to $135 \mathrm{~A}$. Bead-on-plate welds were made on stainless steel plates in the flat position. The thickness of the plates varied from $6 \mathrm{~mm}$ to $4.5 \mathrm{~mm}$, so that the plasma arc passed on the $6 \mathrm{~mm}$ thick plate was not able to produce a keyhole in the samples and on the $4.5 \mathrm{~mm}$ thick plate was able to produce a keyhole by using the above welding parameters, as shown in Fig. 2.

\section{Experimental Results and Time Domain Signature}

Figure 3(A) depicts the sound signal of the plasma arc keyhole welding plotted against time when performing a keyhole mode weld on the stainless steel plate. Figure 3(B) is the reference light signal, which generated a slight increase after about $4.5 \mathrm{~s}$ and a step change on the moment of the presentation of the keyhole.

As can be seen from Fig. 3, the amplitude of the sound signal considerably varies during a welding process. In terms of this signature of the sound signal during welding, the course of the plasma arc welding can be roughly classified into three phases: a non-penetration phase (before $4.5 \mathrm{~s}$ ), a transition phase (from $4.5 \mathrm{~s}$ to $16 \mathrm{~s}$ ) and a keyholeformed phase (after $16 \mathrm{~s}$ ). The magnitude of the sound signal during the non-penetration and the keyhole-formed phases has a more regular pattern. The transition stage, on the other hand, is much more irregular possibly caused by the great oscillation of the weld pool.

As described above, the sound signal includes the information of the status of the weld pool. The sampled sound signal is divided into sections, with $N$ samples in each section. Let $x[n]$ denote the corresponding discrete-time sound

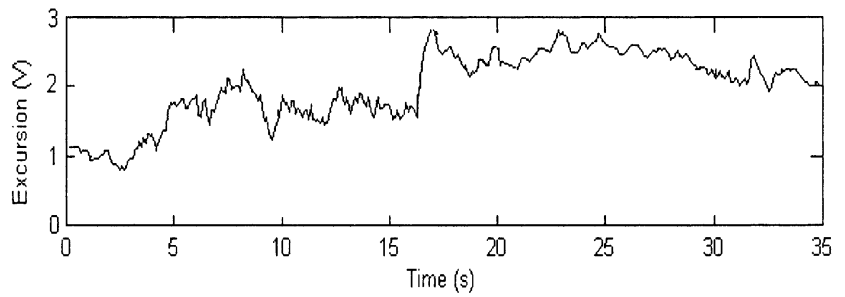

Fig. 4. The amplitude excursion of the acoustic emission.

Table 1. The predetermined threshold in the studied case.

\begin{tabular}{|c|c|c|}
\hline & The excursion & PR algorithm \\
\hline Threshold $\mathrm{T}_{1}$ & $1.2-1.5$ & $2-10$ \\
\hline Threshold $\mathrm{T}_{2}$ & $1.9-2.1$ & \\
\hline
\end{tabular}

signal after the continuous-time sound signal is sampled. The amplitude excursion is calculated for each section and given an index, $k$, defined by the position in the sequence. The amplitude excursion in section $k$ is given by

$$
x_{A}[k]=\sum_{n=(k-1) \cdot N+1}^{k \cdot N}\left|x[n]-\frac{1}{N} \sum_{i=(k-1) \cdot N}^{k \cdot N} x[i]\right|
$$

where $N=2048$, and $k=1,2 \ldots$

Figure 4 shows the result of the amplitude excursion $x_{\mathrm{A}}[k]$ of the sound signal. It can be seen from the figure that the amplitude excursion changed noticeably during welding. On the keyhole-formed phase, the amplitude excursion is larger than that on the transition phase, and much larger than that on the non-penetration phase, which may be related to the plasma jet pulsation at higher frequency band caused by the fluctuation of the arc source voltage. If the predetermined threshold $T_{1}$ and $T_{2}$ are offer (as shown in Table 1), the keyhole states are roughly identified in real time.

\section{Frequency Domain Analysis of the Sound Signal}

\subsection{Sound Power Spectra}

In order to investigate the properties of the sound signal, a frequency domain analysis (using 2048 point FFT) was performed. The typical results of the sound signal power spectra during three different phases are shown in Fig. 5. As can be seen from Fig. 5, the sound power spectra of the three phases appear to be very different each other. There are several dominant peaks in the sound power spectra, such as the peaks at about $2,4,15,17 \mathrm{kHz}$ and so on, in which the $17 \mathrm{kHz}$ dominant peak is the highest. The dominant peaks, which may relate to the plasma jet pulsation, are higher on the keyhole-formed phase than that on the non-penetration phase, and much higher than that on the transition phase. For example, the amplitude of the dominant peak at about $2 \mathrm{kHz}$ is approximately 1100,400 and 60 on the keyhole-formed, non-penetration and transition phases respectively. On the other hand, unlike the higher frequency band, the power spectra of the lower frequency band (less than $300 \mathrm{~Hz}$ ) are not found obvious dominant peaks. The area, below the curve of the power spectral den- 


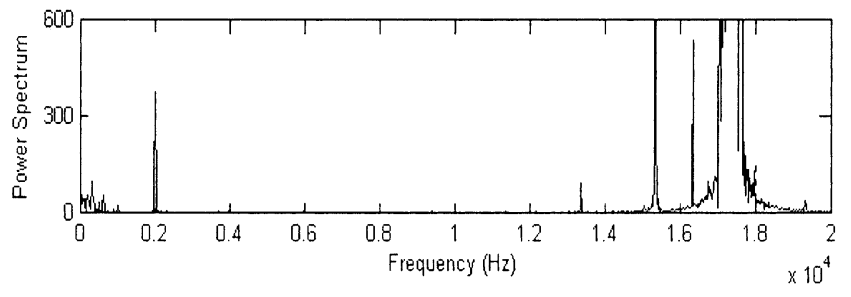

(A)

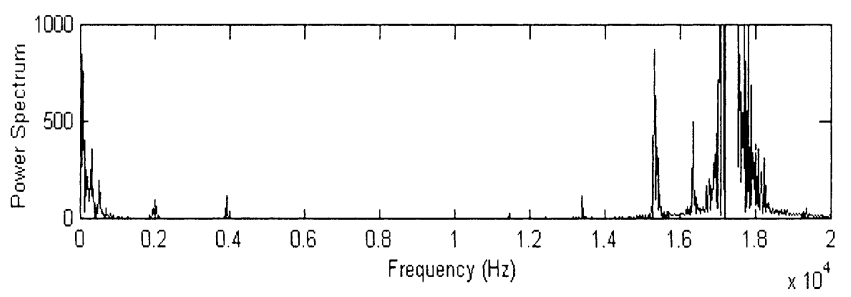

(B)

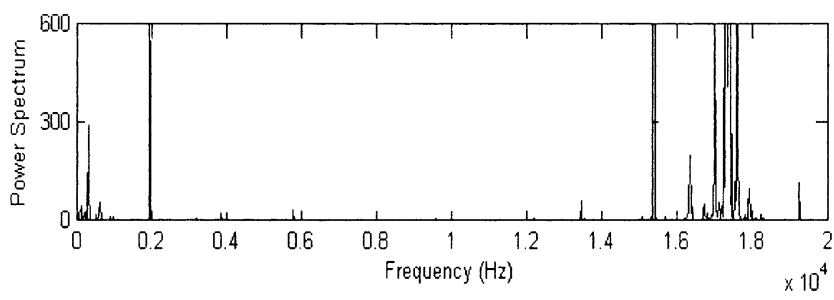

(C)

Fig. 5. The power spectrum of the acoustic emission. A: The non-penetration phase; B: The transition phase; C: The keyhole-formed phase.

sity, of the keyhole-formed phase on the lower frequency band is smaller than that of the non-penetration phase and much smaller than that of the transition phase.

Therefore, the power spectra of the sound signal comprise the information of the keyhole behaviors of plasma arc welding. By means of suitable algorithm, the behaviors of the weld pool of plasma arc keyhole welding can be clearly identified in numerical quantities.

\subsection{The Detection of the Keyhole Status}

As disscussed above, with the variation of the keyhole states the variation trend on the lower frequency band of the sound signal is opposite to that on the higher frequency band, from which the status information of the weld pool can be extracted.

The sound signal is processed by discrete-time windowed Fourier transforms (DWFT). Let $T_{\mathrm{S}}$ denote the sampling rate. By discussion, the analysis-window function is given by

$$
w[n-k]=\left\{\begin{array}{cc}
1 & n=i \cdot k, i \cdot k+1, \ldots, i \cdot k+N-1 \\
0 & \text { otherwise }
\end{array}\right.
$$

where $i$ is the sliding step of the analysis-window, $i=1024$; $N$ is the data points of Fourier transform; $n=0,1,2 \ldots$; $k=0,1,2, \ldots$.

In terms of the DSP (Digital Signal Processing) theory, the discrete-time windowed Fourier transform of $x[n]$ is given by

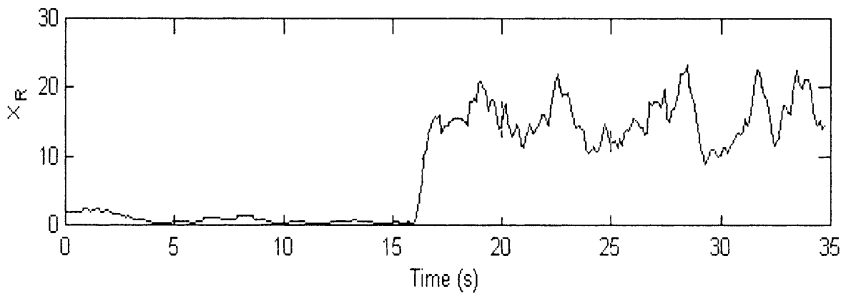

Fig. 6. The result of the PR algorithm.

$$
\begin{aligned}
X_{\text {DWFT }}[k, m] & =\sum_{n=0}^{\infty} x[n] w[n-k] e^{-j \frac{2 \pi}{N} n m} \\
& =\sum_{n=i \cdot k}^{i \cdot k+N-1} x[n] e^{-j \frac{2 \pi}{N} n m} \ldots \ldots \ldots . . .
\end{aligned}
$$

where $m=0,1,2, \ldots, N-1$.

An algorithm of the sound signal, called PR (i.e., Power Ratio) algorithm, is designed as

$$
x_{\mathrm{R}}[k]=\frac{\sum_{m=f_{3} T_{\mathrm{S}} N}^{f_{4} T_{\mathrm{S}} N}\left|X_{\mathrm{DWFT}}[k, m]\right|^{2}}{\sum_{m=f_{1} T_{\mathrm{S}} N}^{f_{2} T_{\mathrm{S}} N}\left|X_{\mathrm{DWFT}}[k, m]\right|^{2}}
$$

where $f_{1}, f_{2}, f_{3}$ and $f_{4}$ are the frequency of the sound signal, $f_{1}=0 \mathrm{~Hz}, f_{2}=200 \mathrm{~Hz}, f_{3}=600 \mathrm{~Hz}$ and $f_{4}=6000 \mathrm{~Hz}$. In fact, the resulting value of the $\mathrm{PR}$ algorithm represents the relative variation of the signal power of the higher frequency band and the lower frequency band.

Figure 6 depicts a typical processing result of the arc signals by the PR algorithm. As can be seen from this figure, the result $x_{\mathrm{R}}$ of the PR algorithm on the keyholeformed phase is larger than that on the other phases, which may result from the natural oscillation of weld pool and the fluctuation of the power source voltage. According to the characteristics, the algorithm can identify the keyhole behaviors of the weld pool if the threshold $T_{1}$ is predetermined.

Under the studied conditions, the predetermined threshold is given in Table 1.

\section{Discussion}

Sound wave is generated by solid vibration and fluid pulsation. During welding, the pulsation of the arc plasma jet, the oscillation of the weld pool and so on are all the sources of the sound signal.

As discussed above, the welding process of the designed samples was divided into three different phases. By observation and comparison with the reference light signal, when the weld process was on non-penetration phase, the weld pool was partial penetration and there was a layer of nonmelted metal below the weld pool. When the weld process was on the transition phase, the weld pool was full penetration, and at this time the output potential of the phototriode slightly increased (as depicted in Fig. 3(B) at about $4.5 \mathrm{~s}$ ), which indicated that the back temperature of the weld became higher. When the weld process was on the keyholeformed phase, part of the arc plasma passed through the 
weld pool and the output potential of the phototriode generated a step change (as shown in Fig. 3(B)). At this time, the metal which is melted in front of the advancing 'keyhole' flows round to the rear where it solidifies to form the weld bead.

The weld pool oscillation is the main source of the sound at lower frequency band. Usually, the natural oscillation frequency of a partial penetration pool is higher than the natural oscillation frequency of a full penetration pool and an abrupt transition occurs between both modes of oscillation. ${ }^{17,18)}$ The free oscillation frequencies for a system consisting of a cylindrical keyhole surrounded by a concentric cylinder of liquid should occur in the $0.5-1 \mathrm{kHz}$ range, ${ }^{19,20)}$ and is the highest among the three phases. As a result, the area below the curve of the low frequency band (less than $200 \mathrm{~Hz}$ ) of the power spectral density is different on the three phases, which is the largest on the transition phase.

The plasma jet pulsation possibly caused by the power source fluctuation and so on is the main source of the dominant peaks of the sound higher frequency band. When the keyhole did not present, the plasma jet acted on the weld pool and the weld pool metal produced cushioning effect on the pulsation of the plasma jet, so that the plasma jet pulsation was suppressed to some extent. This effect was larger on the transition phase than on the non-penetration phase. After the keyhole formed, part of the arc plasma passed through the weld pool and the cushioning effect is decreased, and therefore the amplitude of the plasma jet pulsation became larger. At this time, the plasma jet pulsation may play a dominant role in the acoustic emission, so that the amplitude excursion of the acoustic signal on the keyhole-formed phase is the largest on the three phases (as shown in Fig. 4). The variation trend of the area below the curve of the higher frequency band of the power spectra is opposite to that of the lower frequency band. Therefore, the relative variations of the power spectra of the two bands are able to reflect the behaviors of the weld pool (as depicted in Fig. 6).

Of the two algorithms, the PR algorithm is more reliable to some extent, which may be preferred to use in the detection of the weld quality. Nevertheless, because the required calculation quantity of the PR algorithm is much larger than that of the amplitude excursion, the tradeoff between the calculation time and sensitivity of the algorithm should be considered in practical application.

The algorithms were tested on 105 specimens and have better reliability. However, a suitable change in the threshold value must be adjusted for each piece of equipment and for each type of welding in that the parameters and process factors are usually welding equipment dependent.

\section{Conclusions}

The following conclusions can be drawn from the investigation of the acoustic emission during plasma arc welding:

(1) The sound signal varies with the variation of the status of the weld pool during plasma arc welding.

(2) The low frequency part of the sound signal power spectra is mainly caused by the oscillation of the weld pool. The area below the curve of the low frequency band of the power density spectra is largest on the transition phase.

(3) The high frequency dominant peaks of the sound signal related to the plasma jet pulsation. The amplitude of the dominant peaks is largest on the keyhole-formed phase, the variation trend of which is opposite to that of the area below the curve of the lower frequency band.

(4) The two algorithms are able to detect the keyhole behaviors. The PR algorithm is more reliable than the amplitude excursion although the former spends more data processing time.

\section{REFERENCES}

1) T. Raymond and E. Slatter: Weld. J., 77 (1998), 55.

2) E. Craig: Weld. J., 67 (1988): 19.

3) B. Irving: Weld. J., 76 (1997), 31.

4) U. Dilthey and J. Heidrich: ISIJ Int., 39 (1999), 1067.

5) Y. Suga, T. Shimamura, S. Usui and K. Aoki: ISIJ Int., 39 (1999), 1075.

6) T. P. Quinn and R. B. Madigan: US Patent No. 5,756,967 (1998).

7) S. Adolesson, A. Bahrami, G. Bolmsjo and I. Claesson: Weld. J., 78 (1999), 59-s.

8) R. K. Holbert, R. W. Richardson, D. F. Farson and C. E. Albright: Weld. J., 79 (2000), 89-s.

9) J. M. Vitek: ISIJ Int., 39 (1999), 1088.

10) R. C. Thomson, F. J. Perez-Perez, A. D. Warters and G. Thewlis: ISIJ Int., 39 (1999), 1096.

11) H. D. Steffens and H. Kayser: Weld. J., 51 (1972), 408.

12) J. C. Metcalfe and M. B. C. Quigley: Weld. J., 54 (1975), 401-s.

13) L. F. Martinez, R. E. Marques, J. C. Mcclure and A. C. Nunes: Weld. J., 71 (1992), 49.

14) B. Zheng, H. J. Wang and Q. L. Wang: J. Mater. Process. Technol., 83 (1998), 286

15) M. Futamata, T. Toh, K. Inoue, H. Maruo and Y. Arata: Q. J. Jpn. Weld. Soc., 2 (1984), 727.

16) A. F. Manz: Weld. J., 60 (1981), 23.

17) Y. H. Xiao and G. den. Ouden: Weld. J., 69 (1990), 289-s.

18) Y. H. Xiao and G. den. Ouden: Weld. J., 72 (1993), 428-s.

19) W. W. Duley and Y. L. Mao: J. Phys. D. Appl. Phys., 27 (1994), 1379.

20) J. Kroos, U. Gratzke, M. Vicanek and G. Simon: J. Phys. D. Appl. Phys., 26 (1993), 481. 